\title{
Performance analysis of resource scheduling in LTE femtocell with hybrid access mode
}

\author{
S. Fouziya Sulthana \\ Department of Mechatronics Engineering, SRM Institute of Science and Technology, India
}

\begin{tabular}{|c|c|}
\hline Article Info & ABSTRACT \\
\hline Article history: & \multirow{9}{*}{$\begin{array}{l}\text { Femtocell is a promising technology that intends in solving the indoor } \\
\text { coverage problems so as to enhance the cell capacity. The overall network } \\
\text { performance, in turn depends on the access methods used by the femtocells. } \\
\text { The access method is used to identify about the user's connectivity with the } \\
\text { femtocell network. There are three access mechanisms defined in Third } \\
\text { Generation partnership Project (3GPP) specification for Long Term } \\
\text { Evolution (LTE) femtocells: open, closed and hybrid access mechanisms. } \\
\text { Hybrid access mechanism is mostly preferred by the network for the } \\
\text { effective utilization of resources. But it is important to regulate the proper } \\
\text { scheduling scheme for them. In this paper, scheduling in femtocell is } \\
\text { investigated, where, among the non-subscribers, preference is given to the } \\
\text { users who have high throughput priority metric, thereby increasing overall } \\
\text { throughput of the network. }\end{array}$} \\
\hline Received Jan 4, 2019 & \\
\hline Revised Apr 5, 2019 & \\
\hline Accepted Apr 20, 2019 & \\
\hline Keywords: & \\
\hline Femtocell & \\
\hline LTE & \\
\hline Scheduling & \\
\hline Throughput metric & \\
\hline
\end{tabular}

Copyright $@ 2019$ Institute of Advanced Engineering and Science. All rights reserved.

\section{Corresponding Author:}

S. Fouziya Sulthana,

Departemen Teknik Mekatronika

SRM Institut Sains dan Teknologi

Kattankulathur - 603.203 Tamilnadu, India.

Email: fouziya.s@gmail.com

\section{INTRODUCTION}

There is considerable evolution in the field of mobile technologies over the past decade. With the growing demand of various applications, it is very challenging for a network to enhance the data rate, such that, the Quality of Service (QoS) of all the users are guaranteed with satisfied level. Nowadays, half of the traffic arises from indoor, which undergo various coverage and penetration problems. So, the femtocells, together with the advanced wireless technologies like Long term Evolution (LTE) are considered as emerging solution to overcome these problems, thereby enhancing the cell capacity $[1,2]$

Femtocells (also called Home evolved Node B (HeNB)) are low power nodes that can be overlaid in the existing macro base stations [3]. So, the Third Generation Partnership Project (3GPP) has taken initiatives for the development of two tier macrocell and femtocell networks in LTE - Advanced specifications. The information that is generated from the indoor environment experiences poor quality of signals and severe signal degradation due to high penetration losses. Hence, the femtocells are mainly introduced to provide traffic offloading from macrocells. This traffic offloading causes the requirement of lesser macrocell sites, which results in huge saving of investment expenses and uninterrupted network connectivity to the users, both in outdoor and indoor environments [4].

Femtocells are operated in the licensed frequency spectrum. Since, macrocell and femtocells uses shared frequency bands, the extensive installation of femtocells lead to cross tier interference related issues [5]. The vulnerability of the interference is related to the accessing mechanism used by the femtocell network [6]. In this mechanism, the users who are registered to the femtocell network are called as subscribers and others are called as non subscribers. The access mechanisms that are developed for femtocell networks are shown in Figure 1. 


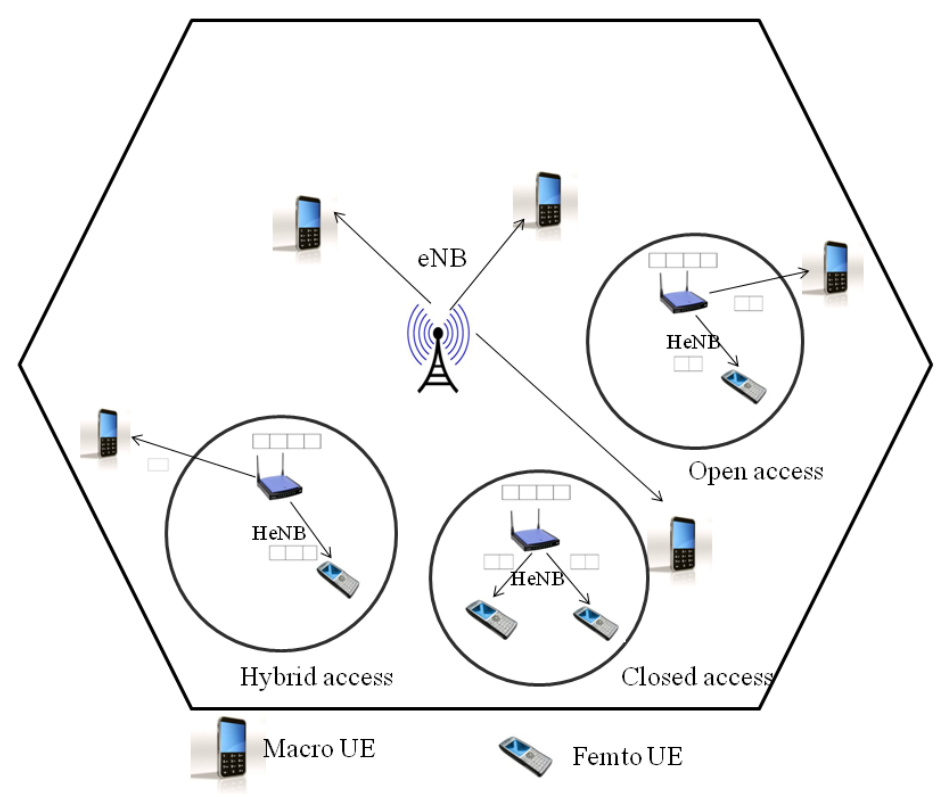

Figure 1. Accessing modes in LTE femtocells

In closed access mode, the subscribers can only be able to access the femtocell network and the non subscribers are provided with strong interference when they come to the vicinity of the network. Many studies related to interference reduction, power control have been done for this closed access modes [7-9]. In open access mode, all the users, irrespective of subscribers and non subscribers can be able to access the network. Though this mode reduces the cross-tier interference, it increases the number of handovers that provide negative impact on the operators. Previous studies have shown that the performance of the system is improved by traffic offloading of this access mode [10-12]. To compromise between these two modes, the hybrid access mode has been introduced, where, all the users are still able to connect to the femtocell network, but priority is given to the subscribed users [13]. Since this mode deals with both the subscribers and non subscribers, scheduling of resources and providing QoS is very difficult to carry out. Proper resource sharing methods should be adopted for the femtocell network because initially it is essential to serve the subscribed users with adequate resources.

Several studies have been carried out regarding resource allocation in femtocell networks. In general, most of the resources scheduling techniques employ First in First Out (FIFO), round robin and proportional fairness algorithms for the allocation of resources among non subscribers [14-16]. In [17], scheduling method in hybrid access femtocell is developed, which provide opportunistic based scheduling to the non subscribers. In [18], a resource allocation scheme is proposed for the femtocells, where, the non subscribers are given prioritized by assigning larger weights to them. Priority based scheduling methods are proposed in [19-22], where, highest preference is given to real time traffic users. Though these methods focus on providing QoS to subscribers, the overall network throughput is not taken into consideration.

In this paper, a scheduling method for LTE femtocell with hybrid access mode is proposed, where, preferential access is given to the non subscribers who have high throughput priority metric value. The rest of the paper is organized as follows: Section 2 describes the system model considered. The proposed scheduling method is explained in Section 3 in detail. Section 4 gives the simulation results and conclusions are drawn in Section 5 .

\section{SYSTEM MODEL}

In this work, a single macrocell is considered in the downlink direction, which contains 1-30 femtocell base stations. The macrocell base station (eNB) is positioned at centre of the cell and the femtocell base stations (HeNB) are distributed randomly inside the cell. It is assumed that the transmit power is evenly distributed among all the subcarriers used. Another assumption is made that the coverage area of the considered femtocell base stations are not overlapping with one another inside the macrocell base station and all base stations are associated with a local module as illustrated in Figure 2. This local module is responsible for on the whole resource management of the system. The local module acquires the channel information 
through the feedback method at the start of every Transmit Time Interval (TTI) and take decision on serving of corresponding base station to the user. TTI is the allocation period in LTE that lasts for $1 \mathrm{~ms}$ duration. It is made assumption that all the femtocell base stations use hybrid access mechanism with limited number of serving of users.

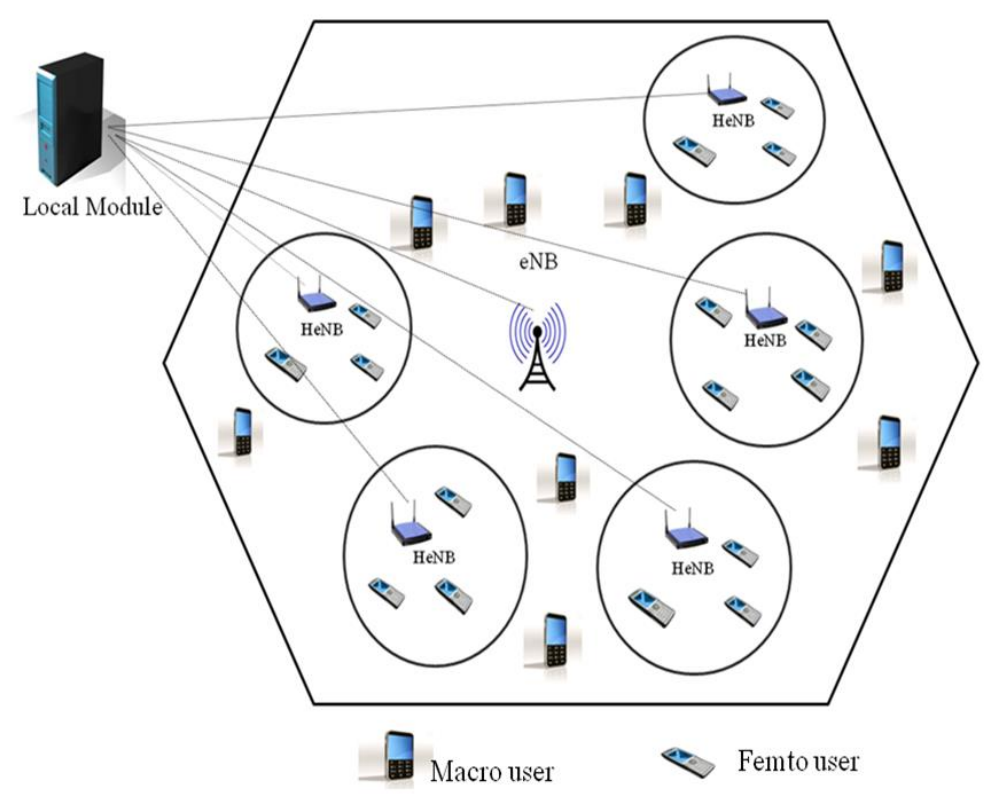

Figure 2. System model

LTE system uses Orthogonal Frequency Division Multiple Access (OFDMA) in the downlink, where the bandwidth is divided into physical resource blocks in time and frequency domain [23]. In this system, if a user has high Signal to Interference Noise Ratio (SINR) from a femtocell than the macrocell to which it is originally associated, then the macrocell can offload the traffic of the user to the femtocell, provided, the femtocell has enough resources. But the subscribers of femtocells are given priority over the non subscribers. The non subscribers will be initially connected to the macrocell base station. As soon as they come in close proximity to the the femtocell coverage area, the local module has to decide whether it should be offloaded to femtocell by analyzing the link condition.

\section{PROPOSED SCHEDULING METHOD}

The radio resources are very much limited in the wireless network environment. So, the effective utilization of resources is very much essential and it is a highly challenging task. The allocation of resources among users needs to be very well controlled. When the users are moving away from the base station, the number of resources to be served to that user increases. So, the hybrid access femtocells play an important role in increasing the effective usage of resources, thereby improving the overall network capacity. The hybrid access femtcell shares some of its resources to the non subscribers.

In the proposed resource scheduling method, the non subscribers who have high throughput priority metric are given preference to access the femtocell. Since the femtocells use hybrid access mechanism, the serving base station has to be selected before scheduling the users. The base station that needs to serve the user is selected by the local module by analyzing the SINR of a user pertaining to macrocell and that of femtocells.

The user's SINR value with reference to macrocell base station $m$ in $n^{\text {th }}$ resource block is found as

$$
\gamma_{m, n}=\frac{P_{m, n} G_{m, n}}{\sum_{f \in F} P_{f, n} G_{f, n}+N_{o} w}
$$


where $P_{m, n}$ and $G_{m, n}$ are the transmit power and channel gain from the macrocell base station, $P_{f, n}$ and $G_{f, n}$ are the transmit power and channel gain from femtocell base stations $f \in F$ in the network, $N_{o}$ is the spectral density of noise and $w$ is the resource block bandwidth.

The user's SINR value with reference to the femtocell base station $k \in F$ in $n^{\text {th }}$ resource block is found as

$$
\gamma_{k, n}=\frac{P_{k, n} G_{k, n}}{P_{m, n} G_{m, n}+\sum_{i \in F \notin k} P_{i, n} G_{i, n}+N_{o} w}
$$

Where $P_{k, n}$ and $G_{k, n}$ are the transmit power and channel gain from femtocell base station $k \in F$ to the user, $P_{i, n}$ and $G_{i, n}$ are the transmit power and gain of the channel from the interfering femtocells to the user.

In the beginning, the user will find out the SINR for all the base stations in the coverage area of the cell. It derives the SINR value from the knowledge of channel information. Accordingly, the modulation order is selected to the user by the base station [24].

After estimating the SINR, the user ensures that the difference between the macrocell $\left(\gamma_{m}\right)$ and its nearest femtocell $\left(\gamma_{f}\right)$ SINR value is greater than the predefined threshold value $\left(\gamma_{T h}\right)$ as given in equation (3). If the variation in SINR value of a user is more than the threshold value then the user may request to access the femtocell network. This may be set according to the network operator's requirement.

$$
\gamma_{m}-\gamma_{f} \geq \gamma_{T h}
$$

For performance analysis it is considered as $7 \mathrm{~dB}$. This is due to the fact that, with this considered value, a user can change from one modulation order to other, which leads to decrease in the requirement of resources, thereby improving the effective utilization. The equation (3) is estimated merely for the non subscribers. The femtocell network can be connected to the non subscribers by analyzing the throughput priority metric of the user and available resources. The procedure followed at the local module for linking the user to the femtocell network is given in Algorithm I.

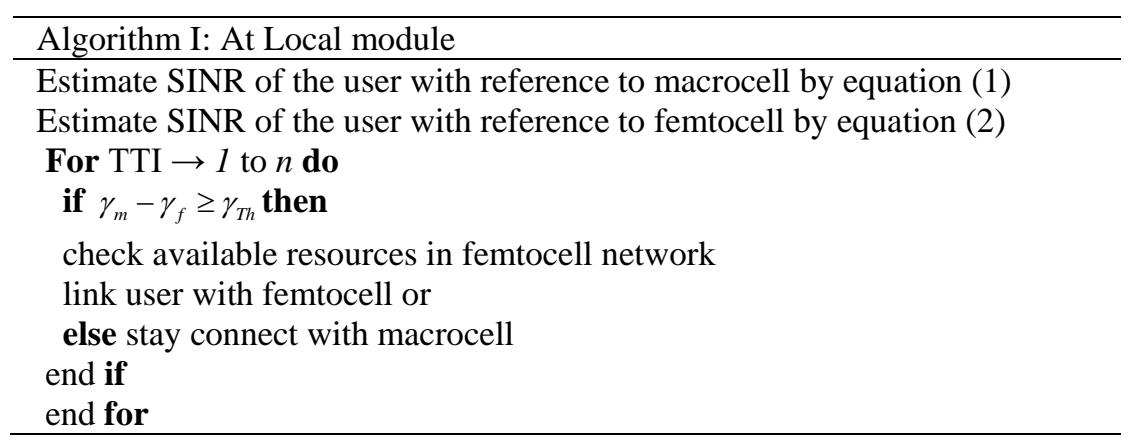

In hybrid access mechanism, different allocation algorithms may be used for subscribers and non subscribers. The conventional technique of allocating resources to the non subscribers is FIFO because of its simple flow of structure. But, by giving priority to the users who have high throughput value, the overall throughput of the network can be improved. The three-level scheduling algorithm that is used centrally at the macrocell base station [25] is used in femtocells also for scheduling the non subscribers. This algorithm aims in improving overall cell throughput while maintaining the fairness among users.

With three level scheduling method, the users who are in good channel conditions are given preference both in macrocell and femtocell. In femtocell network, after guaranteeing minimum rate requested by the subscribers, the remaining resources are scheduled to non subscribers. While doing scheduling to the non subscribers, preference is given to the users who have high CQI value to increase the overall throughput of total users. For simplicity, only throughput priority metric is calculated for non subscribers while doing scheduling in femtocells.

In the femtocell network, the resources are predetermined for subscribers and non subscribers but it is necessary to guarantee the minimum data rate demanded by the subscribers. While doing scheduling to the non subscribers preference is given to the users with high Channel Quality Indicator (CQI) value. 
By using three level algorithms, the throughput priority metric is estimated which is given as

$$
T_{k}=\frac{R_{k, n}}{R_{k, a v g}}
$$

where $R_{k, n}$ is the data rate achieved by $k^{\text {th }}$ user in $n^{\text {th }}$ resource block and $R_{k, a v g}$ is the average data rate achieved by $k^{\text {th }}$ user. The non subscribers who have throughput priority metric greater than threshold value are chosen for allocation. The procedure followed at the femtocells for sharing resources with the non subscribers is given in Algorithm II.

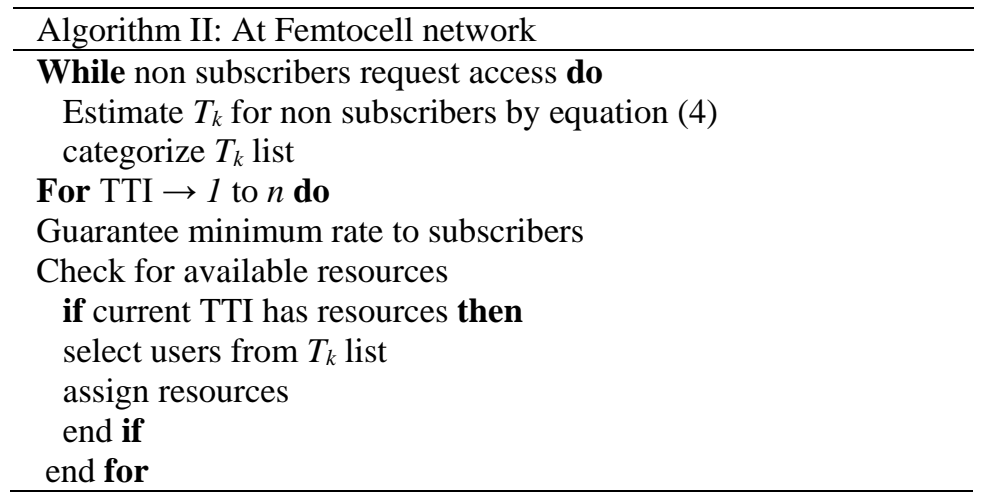

\section{SIMULATION RESULTS}

An LTE downlink scenario is considered with single macrocell base station in which femtocells are randomly distributed within the coverage area of the cell without overlapping to each other. For simulation purpose, the assumption is made that the users inside the cell are moving with a constant speed of 3 and 30 $\mathrm{kmph}$, and the local module receives perfect channel quality report from the user. To minimize the complexity, it is taken into consideration that at any point of time there will be $60 \%$ of real time service users and $40 \%$ of non real time service users. Real time service includes video and Voice over Internet Protocol (VoIP) traffic and non real time service users include Best Effort (BE) traffic. Video traffic with source rate $128 \mathrm{kbps}$ are produced by H.264 trace-based coder and VoIP traffic with source rate of $8 \mathrm{kbps}$ are generated from G.729 audio coder. BE traffic is produced by ideal source which has always packets to send. The main simulation parameters are shown in Table 2.

Table 2. The main simulation parameters

\begin{tabular}{ll}
\hline Parameter & Value \\
\hline Bandwidth & $10 \mathrm{MHz}$ \\
Carrier frequency & $2 \mathrm{GHz}$ \\
Subcarrier bandwidth & $15 \mathrm{kHz}$ \\
Macrocell radius & $500 \mathrm{~m}$ \\
Femtocell radius & $15 \mathrm{~m}$ \\
Macrocell transmit power & $43 \mathrm{dBm}$ \\
Femtocell transmit power & $17 \mathrm{dBm}$ \\
Number of femtocell network & $1-30$ \\
Maximum number of mobile users & 300 \\
Maximum subscribers per femtocell & 4 \\
Minimum throughput of subscriber & $4 \mathrm{Mbps}$ \\
Macrocell user's speed & $3 \mathrm{kmph}$ and 30 kmph \\
Femtocell user's speed & $0-1 \mathrm{kmph}$ \\
Channel model & $6-\mathrm{Ray}$ Typical Urban (TU) for outdoor WINNER II for indoor \\
Modulation order & QPSK, 16 QAM, 64 QAM \\
Wall attenuation & $20 \mathrm{~dB}$ \\
Simulation duration & $100 \mathrm{~s}$ \\
\hline
\end{tabular}

The performance of the proposed resource allocation method is analyzed in terms of throughput of non subscribers and total users in the system. The analysis is done by incrementing the number of femtocell network in the macrocell region. For performance analysis, LTESim, an open source simulator is used [26]. The average throughput of non subscribers at $3 \mathrm{kmph}$ and $30 \mathrm{kmph}$ is shown in Figure 3 . From the figure it is 
observed that the average throughput of non subscribers is improved when compared with the conventional FIFO based allocation method. This is because the proposed scheduling scheme provides resource allocation based on throughput priority metric. So, the non subscribers who have high CQI value are given priority thus increasing the average throughput of them. Whereas, the conventional method allocates on first come first serve basis irrespective of their channel condition.

However, there is reduction in the average throughput of non subscribers when there is increase in the speed. This is due to the fact that, when the speed of the non subscribers increases, there will be high possibility of loss of the packets that are send from the base station. By using the proposed scheduling method there is $5 \%$ and $11 \%$ increase in the average throughput of non subscribers when compared with the already existing conventional FIFO based allocation method for the taken simulation parameters at $3 \mathrm{kmph}$ and $30 \mathrm{kmph}$, respectively.

The average throughput of total users is depicted in Figure 4. Since the users who have good channel conditions are given priority in both macrocell and femtocell, the average throughput of all the users in the system is improved when compared with the conventional FIFO based scheduling method. The proposed method shows $10 \%$ and $9 \%$ increase in the average throughput of total users at $3 \mathrm{kmph}$ and $30 \mathrm{kmph}$, respectively when compared with the conventional method of scheduling.

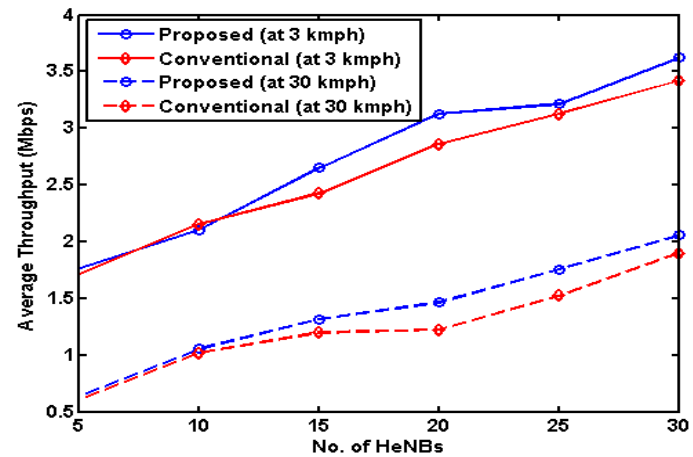

Figure 3. Average throughput of non subscribers compared to conventional method of scheduling

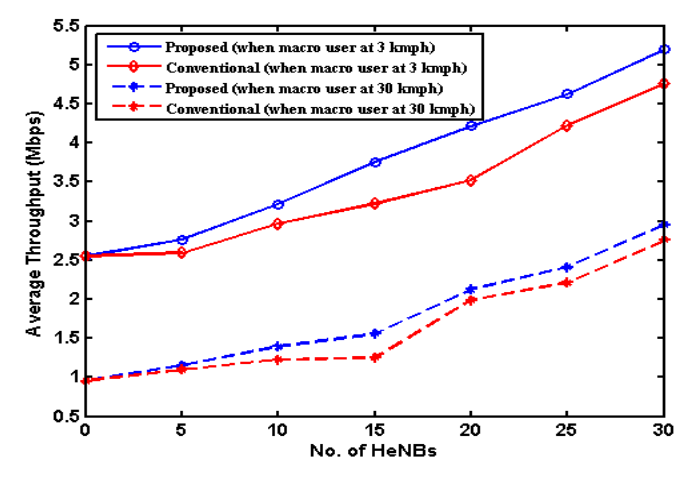

Figure 4. Average throughput of total users compared to conventional method of scheduling

The average throughput of non subscribers with respect to other access mechanisms is given in Figure 5 (a) and (b). From the figure it is clear that non subscriber has highest throughput when they use the hybrid access mechanism. Generally, the non subscribers who are very far from the macrocell base station will try to get access from the nearby femtocell network, provided the femtocell uses hybrid access mechanism. Mostly, they will experience the degraded quality of signals. So, more resources are necessary for them to satisfy their QoS. Alternatively, when they are connected to the femtocell network with hybrid access mode, the resources are efficiently utilized, thereby increasing the average throughput of non subscribers.

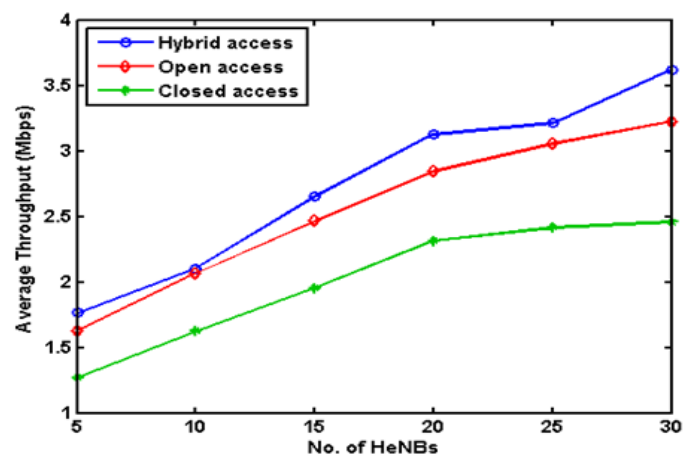

(a)

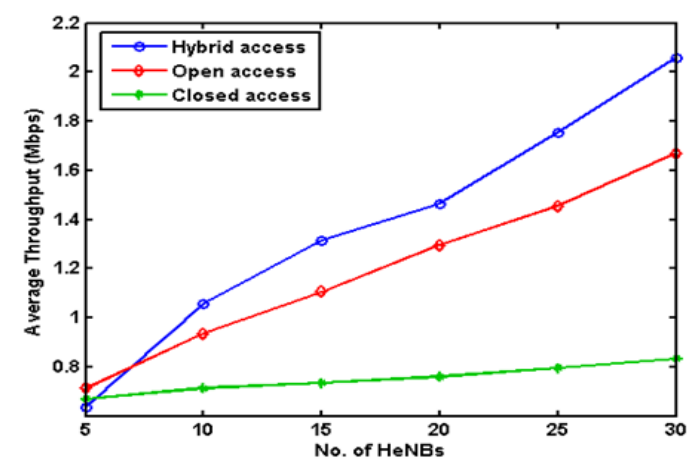

(b)

Figure 5. Average throughput of non subscribers compared to other accessing mechanisms (a) at $3 \mathrm{kmph}$ and (b) at $30 \mathrm{kmph}$ 
In femtocells with open access mechanism, the network allows all the non subscribers to get connected when they come near the vicinity of the network. This leads to increase in the number of handovers since the users are moving with a considerable speed thereby decreasing the overall throughput of the non subscribers. With same environment, the femtocells with closed access mechanism allow only the subscribed users to get connected to the network. When the non subscribers come near the vicinity of the femtocell network they experience a very strong interference from the femtocells. And also, they can get access only from the macrocell base station even if they are transmitting information with degraded quality. As a result, the average throughput of non subscribers in femtocells with closed access mechanism is very much reduced when comparing with other access mechanisms.

The average throughput of total users is analyzed in three different accessing mechanisms with two different speeds and is shown in Figure 6. It is clearly visible that as the speed of the users increases there is decrease in the average throughput because of the packet losses. And also, it is shown that the performance of the femtocells with closed access mechanism is slightly superior when compared to other access mechanisms. This is due to the fact that in closed access mechanism, the femtocell intends to gaurantee the subscriber's individual average throughput by using all of its available resources, thereby increasing the overall average throughput of total users in the system. This may be an advantageous mechanism from the network point of view, but fails in guaranteeing QoS of all the users in the network.

Whereas, in hybrid access mechanism, after guaranteeing subscriber's minimum resource requirement, some resources are reserved for non subscriber's utilization. This increases the user's average throughput because of the effective utilization of resources but not as much of closed access mechanism. On the other hand, open access mechanism shows poor performance because of increase in signaling and number of handovers.

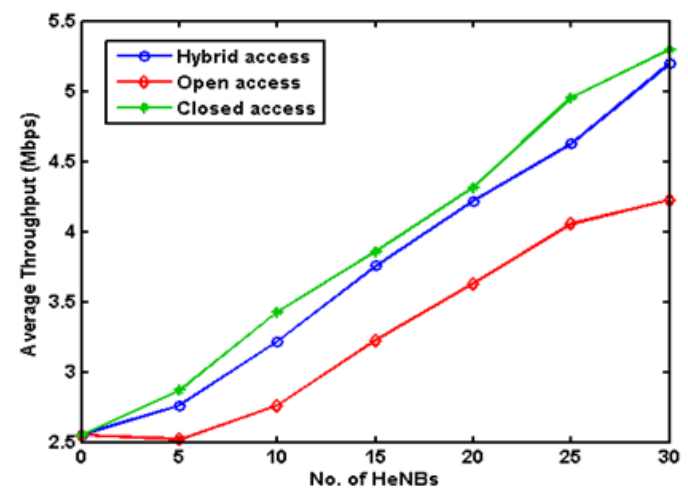

(a)

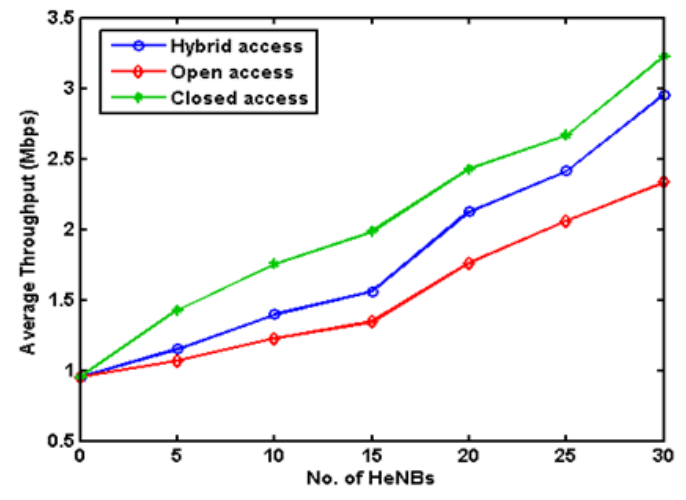

(b)

Figure 6. Average throughput of total users compared to other accessing mechanisms (a) at $3 \mathrm{kmph}$ and (b) at $30 \mathrm{kmph}$

\section{CONCLUSION}

In this paper, scheduling for hybrid access femtocell is investigated, which gives preference to the users who have high throughput priority metric. Since hybrid access mechanism is used in the femtocells, the serving base station is selected before scheduling the users. Local module in the network is responsible for selecting base station to the users based on their SINR feedback. After selecting the base station, scheduling is done to the non subscribers who have high throughput priority metric. By using this proposed method, the average throughput of the total users in the system is increased. There is $10 \%$ and $9 \%$ increase in the average throughput of total users at $3 \mathrm{kmph}$ and $30 \mathrm{kmph}$, respectively when compared with the conventional method of scheduling.

\section{REFERENCES}

[1] Vikram Chandrasekhar, Jeffrey G. Andrews and Alan Gatherer, "Femtocell Networks: A Survey," IEEE communication magazine, vol. 46, no. 9, pp. 59-67, Sep 2008.

[2] H Claussen, LTW Ho, JG Andrews, "An overview of the femtocll concept," Bell Labs Tech Journal, vol. 13, no. 1, pp. 224-246, 2008. 
[3] Alan Barbieri, Aleksandar damnjanovic, Tingfang Ji, Juan Montojo, Yongbin Wei, Durga Malladi, Osok Song and Gavin Horn, "LTE Femtocells: System design and performance analysis," IEEE journal on selected areas in communications, vol. 30, no. 3, pp. 586-593, Apr 2012.

[4] D Calin, H Claussen, H Uzunalioglu, "On femto deployment architectures and macrocell offloading benefits in joint macro-femto deployments," IEEE communication magazine, vol. 48, no. 1, pp. 26-32, 2010.

[5] P Lin, J Zhang, Y Chen, Q Zhang, "Macro-femto heterogeneous network deployment and management: from business models to technical solutions," IEEE wireless communication, vol. 18, no. 3, pp. 64-70, 2011.

[6] Han-Shin Jo, Ping Xia and Jeffrey G Andrews, "Open, closed and shared access femtocells in the downlink," Eurasip journal on wireless communications and Networking, vol. 2012, no. 363, pp. 1-16, 2012.

[7] P Mach, Z Becvar, "QoS guaranteed power control mechanism based on the frame utilization for femtocells," EURASIP journal of wireless communication and networking, vol. 2011, no. 16, 2011.

[8] Liqun Fu, Soung Chang Liew and Jianwei Huang, "Fast algorithms for joint power control and scheduling in wireless networks," IEEE Transactions on Wireless Communications, vol. 9, no.3, pp. 1186-1197, 2010.

[9] Iturralde M., Yahiya T. A., Wei A. and Beylot, A., "Interference Mitigation by Dynamic Self Power Control in Femtocell Scenarios in LTE Network," IEEE Global Communications Conference, Anaheim, pp. 1-6, 2012.

[10] Xia P., Chandrasekhar V. and Andrews J. G. "Femtocell Access Control in the TDMA/OFDMA Uplink," IEEE Global Telecommunications Conference, Miami, USA, pp. 1-5, 2010.

[11] Jo H. S., Xia P. and Andrews J. G. "Downlink Femtocell Networks: Open or Closed?," IEEE International Conference on Communications, Kyoto, Japan, pp. 1-5, 2011.

[12] L. Li, C. Xu, M. Tao, "Resource allocation in open access OFDMA femtocell networks," IEEE Wireless Communication Letters, vol. 1, no. 6, pp. 625-628, 2012.

[13] Marshoud H., Otrok H., and Barada H., "Macrocell Femtocells Resource Allocation with Hybrid Access Motivational Model," Physical Communication, vol. 11, pp. 3-14, 2014.

[14] Y. L. Lee, T. C. Chuah, J. Loo and A. Vinel, "Recent advances in radio resource management for heterogeneous LTE/LTE-A networks," IEEE Commun. Surv. Tuts., vol. 16, no. 4, pp. 2142-2180, Jun 2014.

[15] A. Hatoum, R. Langar, N. Aitsaadi, R. Boutaba and G. Pujolle, "Cluster based resource management in OFDMA femtocell networks with QoS guarantees," IEEE Trans. Veh. Technol., vol. 63, no. 5, pp. 2378-2391, Jun 2014.

[16] de la Roche G., Valcarce A., Lopez-Perez D. and Jie Zhang. "Access Control Mechanisms for Femtocells," IEEE Communications Magazine, vol. 48, no. 1, pp. 33-39, 2010.

[17] L. B. Le, D. Niyato, E. Hossain, D. I. Kim and D. T. Hoang, "QoS-aware and energy-efficient resource management in OFDMA femtocells," IEEE Trans. Wirel. Commun., vol. 12, no. 1, pp. 180-194, Jan 2013.

[18] R. Estrada, A. Jarray, H. Otrok, Z. Dziong and H. Barada, "Energyefficient resource allocation model for OFDMA macrocell/femtocell networks," IEEE Trans. Veh. Technol., vol. 63, no. 5, pp. 3492-3437, Sep 2013.

[19] R. Balakrishnan and B. Canberk, "Traffic-aware QoS provisioning and admission control in OFDMA hybrid small cells," IEEE Trans. Veh. Technol., vol. 63, no. 2, pp. 802-810, Feb 2014.

[20] H. Zhang, C. Jiang, N. C. Beaulieu, X. Chu, X. Wen, M. Tao, "Resource Allocation in Spectrum-Sharing OFDMA Femtocells with Heterogeneous Services," IEEE Trans. Commun., vol. 62, no. 7, pp. 2366-2377, Jul 2014.

[21] Y. L. Lee, J. Loo and T.C. Chuah, "Dynamic Resource Management for LTE based Hybrid Access Femtocell System," IEEE Systems Journal, vol. 12, no. 1, pp. 959-970, Mar 2018.

[22] S. Fouziya Sulthana and R. Nakkeeran, "Priority based resource scheduling in hybrid accessLTE femtocell," Journal of communications and Information Networks, vol. 3, no. 2, Jun 2018.

[23] 3GPP, "Tech. Specific. Group Radio Access Network - Physical Channel and Modulation (Release 8)," 3GPP TS 36.211.

[24] Fan J., Yin Q., Li G.Y., Peng B., Zhu X., "MCS Selection for Throughput Improvement in DL LTE System," IEEE conference on Computer Communications and Networks, Hawaii, pp. 1-5, 2011.

[25] S. Fouziya Sulthana and R. Nakkeeran, "A Simple Three Level Downlink Packet Scheduling to Improve Throughput in LTE Network," IEICE Communication Express, vol. 4, no. 9, pp. 293-298, Sep 2015.

[26] Piro G., Grieco L. A., Boggia G., Capozzi F., Camarda P., "Simulating LTE Cellular Systems: An Open Source Framework," IEEE Transactions on Vehicular Technology, vol. 60, no. 2, pp. 498-513, 2010.

\section{BIOGRAPHIES OF AUTHORS}

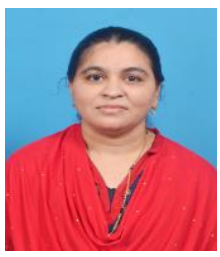

Dr. S. Fouziya Sulthana received her B. E degree in Electronics and Communication from Bharadidasan University, Tamilnadu in 2002 and $\mathrm{M}$. Tech and $\mathrm{Ph}$. D in Electronics and Communication from Pondicherry University, Puducherry in 2008 and 2017, respectively. She has worked in many private engineering colleges since 2008 and published many papers in refereed journals and conferences. Her current research focuses on Radio Resource Management in broadband networks. 